\title{
A new total synthesis of natural isocoumarin, thunberginol $B$
}

\author{
Ghulam Qadeera, Nasim Hasan Rama ${ }^{a}$, and Syed Jabbar Hussain Shah ${ }^{\mathrm{b}}$ \\ ${ }^{a}$ Department of Chemistry, Quaid-I-Azam University, Islamabad 45320, Pakistan \\ ${ }^{b}$ Institute of Organic Chemistry, Humbolt University, Brooke-taylor-strasse 2, 12489 Berlin, \\ Germany \\ E-mail: nasimhrama@yahoo.com
}

\begin{abstract}
3,5-Dimethoxyhomophthalic acid is a key intermediate for the synthesis of highly biological active naturally and non-naturally occurring isocoumarins and 3,4-dihydroisocoumarins. It was synthesized efficiently in five steps from 3,5-dimethoxybenzaldehyde (1) via a series of reactions including synthesis of 3,5-dimethoxycinnamic acid (2) and 3-(3',5'-dimethoxyphenyl)propionic acid (3), cyclization of 3-(3',5'-dimethoxyphenyl)propionic acid (3) to 5,7-dimethoxy-1indanone (4) and oxidative decomposition of methyl 2-hydroxy-2-(5,7-dimethyoxy-1-oxo-1Hinden-2(3H)-ylidene)acetate (5) to 3,5-dimethoxyhomophthalic acid (6). Natural isocoumarin, thunberginol B [3-(3',4'-dihydroxyphenyl)-6,8-dihydroxyisocoumarin] (10) was synthesized by the condensation of 3,5-dimethoxyhomophthalic acid (6) with 3,4-dimethoxybenzoic acid (7) followed by demethylation of intermediate [3-(3',4'-dimethoxyphenyl)-6,8dimethoxyisocoumarin] (9). The synthesized compounds were purified by HPLC and characterized by elemental analysis, IR, ${ }^{1} \mathrm{H}$ NMR and mass spectrometry.
\end{abstract}

Keywords: 3,5-Dimethoxyhomophthalic acid, thunberginol B, synthesis, demethylation

\section{Introduction}

Isocoumarin structures are important components in many natural products that exhibit a broad range of biological activitities including antiallergic and antimicrobial, ${ }^{1,2}$ immunomodulatory, ${ }^{3}$ cytotoxic, ${ }^{4}$ antifungal, ${ }^{5}$ antiinflammatory, ${ }^{6}$ antiangiogenic, ${ }^{7}$ anticalmodulin-sensitive cyclic guanosine 30,50-monophosphate phosphodiesterease ${ }^{8}$ effects, and differentiation inducing activity against leukemic cells. ${ }^{9}$ Isocoumarins ${ }^{10}$ are also useful intermediates in the synthesis of a variety of important compounds including some isoquinoline alkaloids. ${ }^{11}$ Thus, a number of methods have been reported in the literature for the synthesis of isocoumarins. ${ }^{12}$ For example, in the last 10 years 3-substituted isocoumarins with no substituent at the 4-position have been synthesized either by a variety of traditional approaches ${ }^{13}$ or by utilizing transition metal 
catalyzed reactions. ${ }^{14}$ The synthesis of 3,4-disubstituted isocoumarins has received considerable attention too ${ }^{6,15}$ and, recently, a number of these compounds have been prepared in good yields via palladium-catalyzed annulation of internal alkynes. ${ }^{15 \mathrm{~d}}$ However, this last catalytic method is somewhat limited in synthetic scope since it is highly regioselective only for symmetrical disubstituted acetylenes containing a highly sterically demanding group in the position adjacent to the carbon-carbon triple bond.

Thunberginol B, a natural isocoumarin derivative, was isolated from Hydrangeae Dulcis Folium, the fermented leaves of Hydrangea macrophylla SERINGE var. thunbergii MAKINO, as antiallergic constituents. ${ }^{16-19}$ Thunberginol B (10) inhibited the histamine release from rat peritoneal mast cells induced by compound 48/80, calcium ionophore $\mathrm{A} 23187$, with $\mathrm{IC}_{50}$ values of less than $100 \mu \mathrm{M}$. Structure-activity studies have shown that isocoumarins such as thunberginol $\mathrm{A}$ and $\mathrm{B}$ are more potent as histamine release inhibitors as compared to the dihydroisocoumarins such as hydrangenol, thunberginol D and E. ${ }^{18-20}$ In addition, these thunberginols showed antimicrobial activity against oral bacteria. ${ }^{18}$

Due to their biological importance, various routes were adopted to synthesize these compounds. ${ }^{21-22}$ We wish to report here a new convenient route for the synthesis of Thunberginol B.

\section{Results and Discussion}

We now disclose a short and efficient synthesis of thunberginol B 10 (Scheme 1). Key intermediate 3,5-dimethoxyhomophthalic acid (6) was prepared from commercially available 3,5-dimethoxybenzaldehyde (1), which on condensation with malonic acid in the presence of dry pyridine and piperidine yielded 3,5-dimethoxycinnamic $\operatorname{acid}^{23,24}$ (2), which showed a characteristic broad singlet at $\delta 11.7$ for $-\mathrm{OH}$ in its ${ }^{1} \mathrm{H}-\mathrm{NMR}$ spectrum and $\mathrm{C}=\mathrm{C}$ absorption at $1600 \mathrm{~cm}^{-1}$ in its IR spectrum. The mass spectrum of this compound showed a molecular ion peak at $\mathrm{m} / \mathrm{z}$ 208, which agreed with its molecular weight. 3,5-Dimthoxycinnamic acid (2), on reduction with sodium amalgam, yielded 3-(3',5'-dimethoxyphenyl)propionic acid (3), which exhibited a broad singlet at $\delta 11.66 \mathrm{ppm}$ for $\mathrm{COOH}$ in its ${ }^{1} \mathrm{H}-\mathrm{NMR}$ spectrum, which is exchangeable with $\mathrm{D}_{2} \mathrm{O}$. The carboxylic carbonyl absorption in its IR spectrum was observed at $1722 \mathrm{~cm}^{-1}$. The mass spectrum of this propionic acid 3 showed a molecular ion peak at $\mathrm{m} / \mathrm{z}$ of 210 and a characteristic peak at m/z $165\left[\mathrm{M}^{+}-\mathrm{COOH}\right]$. 3-(3',5'-Dimethoxyphenyl)propionic acid (3), on cyclization with polyphosphoric acid, furnished 5,7-dimethoxy-1-indanone (4) and its structure was confirmed by two $3 \mathrm{H}$ singlets for $\mathrm{OCH}_{3}$ at $3.80 \mathrm{ppm}$ and $3.77 \mathrm{ppm}$ in the ${ }^{1} \mathrm{H}-\mathrm{NMR}$ spectrum, while carbonyl absorption in the IR spectrum appeared at $1685 \mathrm{~cm}^{-1}$. The mass spectrum of the indanone 4 showed a molecular ion peak at m/z 192. 5,7-Dimethoxy-1-indanone (4) on reaction with diethyl oxalate in the presence of sodium methoxide in dry benzene gave methyl (2,3-dihydro-5,7-dimethoxy-1-oxo-1H-indene-2-ylidene)hydroxy acetate (5) and the structure of the latter was confirmed by a $3 \mathrm{H}$ singlet for -COOMe at $3.95 \mathrm{ppm}$ in its ${ }^{1} \mathrm{H} \mathrm{NMR}$ 
spectrum. The IR spectrum of this ester showed two additional bands at $3600-3000 \mathrm{~cm}^{-1}$ for OH and $1744 \mathrm{~cm}^{-1}$ for the carbonyl of the ester moiety.
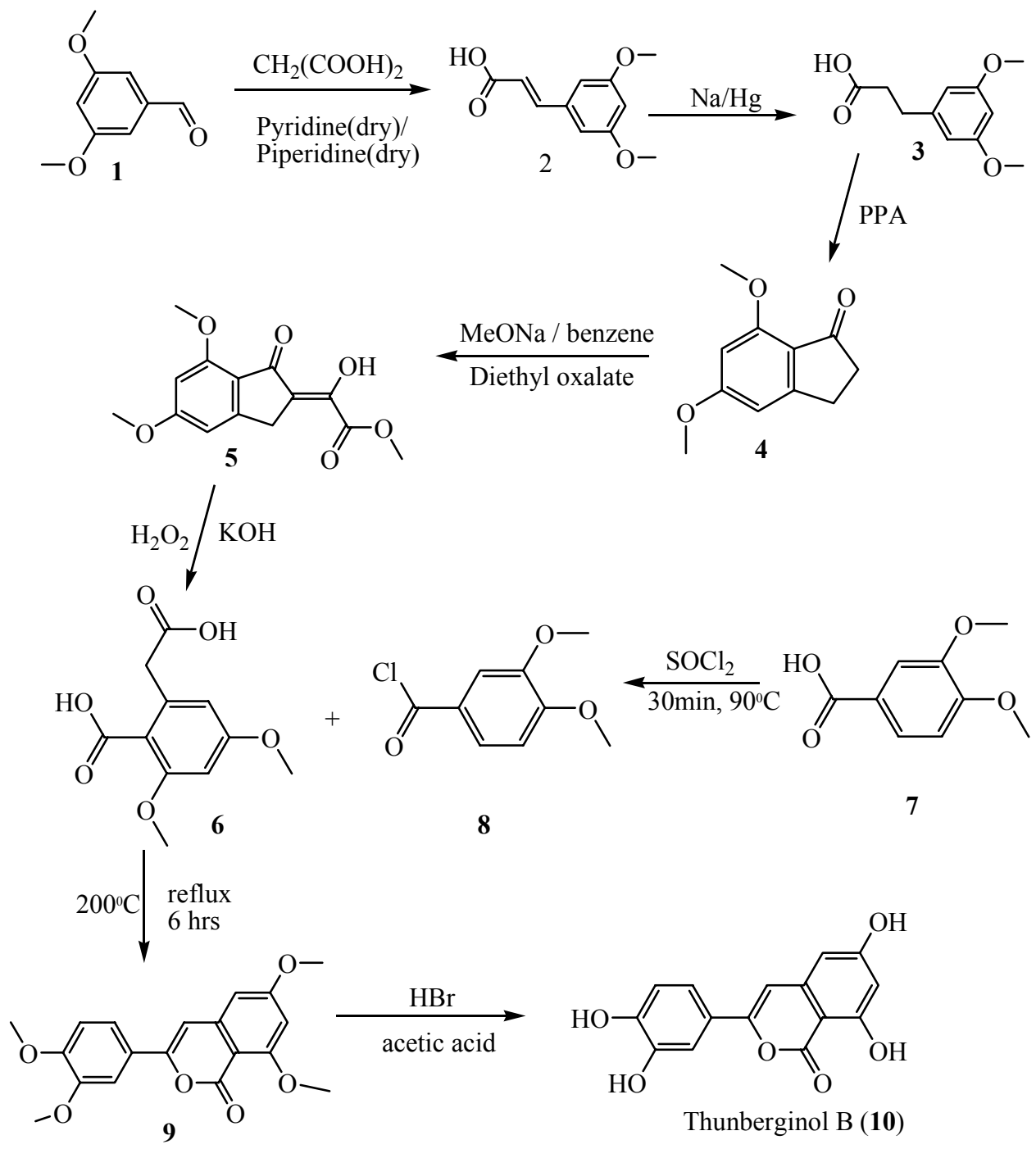

\section{Scheme 1}

The mass spectrum of 5 showed a molecular ion peak at m/z 278. Methyl 2-hydroxy-2-(5,7dimethyoxy-1-oxo-1H-inden-2(3H)-ylidene)acetate (5), on oxidation with hydrogen peroxide in the presence of potassium hydroxide, furnished 3,5-dimethoxyhomophthalic acid (6), which showed a characteristic broad singlet at $\delta 11.2 \mathrm{ppm}$ exchangeable with $\mathrm{D}_{2} \mathrm{O}$ for $-\mathrm{OH}$. The two absorption bands for the carbonyl groups appeared at 1748 and $1697 \mathrm{~cm}^{-1}$ in the IR spectrum. The mass spectrum of this compound showed a molecular ion peak at $\mathrm{m} / \mathrm{z} 240$, which agreed with its molecular weight. The mass spectrum also showed two characteristic peaks at $\mathrm{m} / \mathrm{z}=222$, $195\left[\mathrm{M}^{+}-\mathrm{H}_{2} \mathrm{O}\right.$, - $\left.\mathrm{COOH}\right]$. 3,4-Dimethoxybenzoylchloride (8) was prepared from 3,5dimethoxybenzoic acid (7) on reaction with thionyl chloride. Direct condensation of 3,4- 
dimethoxybenzoylchloride (8) with 3,5-dimethoxyhomophthalic acid (6) at $200^{\circ} \mathrm{C}$ afforded 3(3',4'-dimethoxyphenyl)-6,8-dimethoxyisocoumarin (9). This isocoumarin 9 was purified by HPLC. It showed lactonic carbonyl absorption at $1716 \mathrm{~cm}^{-1}$ and $\mathrm{C}=\mathrm{C}$ absorption at $1599 \mathrm{~cm}^{-1}$ in its IR spectrum and a characteristic $\mathrm{C} 4-\mathrm{H}$ singlet at $\delta 7.0 \mathrm{ppm}$ in its ${ }^{1} \mathrm{H}$ NMR spectrum. Complete demethylation of this isocoumarin 9 with hydrobromic acid (48\%) in acetic acid gave 3-(3',4'-dihydroxyphenyl)-6,8-dihydroxyisocoumarin (10). The IR spectrum of the latter showed a broad absorption at $3386-3360 \mathrm{~cm}^{-1}$ for the phenolic $\mathrm{OH}$ group. In the ${ }^{1} \mathrm{H}$ NMR spectrum, the signal at $\delta 3.83$ ppm for the methoxy groups in 9 had disappeared.

\section{Experimental Section}

General Procedures. All reagents and solvents were commercially available and used as supplied. The melting points of the compounds were determined in open capillaries using a Gallenkamp melting point apparatus and are uncorrected. The infrared spectra were recorded on a Hitachi model 270-50 spectrophotometer as $\mathrm{KBr}$ disks or as neat liquids. ${ }^{1} \mathrm{H}-\mathrm{NMR}$ and ${ }^{13} \mathrm{C}$ NMR (300 MHz) spectra were recorded on a Bruker AM-300 in $\mathrm{CDCl}_{3}$ solution using TMS as an internal standard. HPLC-MS analyses were performed HPLC-Anlage Waters 2695 Alliance, Säule Phenomenex Luna $3 \mu$ Phenyl-Hexyl $(2 \times 150 \mathrm{~mm})$, mobile Phase Water/Acetonitrile (0:100 bis 50:50), Fluss: $0.2 \mathrm{ml} / \mathrm{min}$, Photodiode array detector Waters 996, MS-Detector Waters ZQ2000.. In the mass detector, the fragmenter operated at $70 \mathrm{eV}$. Elemental analysis was performed on a LECO CHNS-932 instrument.

3,5-Dimethoxycinnamic acid (2). 3,5-Dimethoxybenzaldehyde (1) (17g, 0.1mole), molanic acid $(51 \mathrm{~g})$, dry pyridine $(183.6 \mathrm{ml})$ and piperidine $(40.2 \mathrm{ml})$ were heated on boiling water bath for $4 \mathrm{~h}$. The reaction mixture was cooled and poured into cold water $(500 \mathrm{ml})$ and carefully acidified with dilute hydrochloric acid (1:1) with stirring. On filtration white ppt. of 3,5-dimethoxycinnamic acid (2) was obtained (18.3g, 0.08 mole, 86\%). m.p $170-171{ }^{\circ} \mathrm{C}$. IR $\left(v_{\max }, \mathrm{KBr}, \mathrm{cm}^{-1}\right)=3400$ 3200, 2950, 2835, 1685, 1600,1365, 1310. ${ }^{1} \mathrm{H}-\mathrm{NMR}\left(\mathrm{CDCl}_{3}, 300 \mathrm{MHz}\right): \delta(\mathrm{ppm})=6.66(2 \mathrm{H}, \mathrm{d}, J$ $=2.1 \mathrm{~Hz}), 6.5(1 \mathrm{H}, \mathrm{t}, J=2.2 \mathrm{~Hz}), 7.69(1 \mathrm{H}, \mathrm{d}, J=15.5 \mathrm{~Hz}), 6.4(1 \mathrm{H}, \mathrm{d}, J=15.8 \mathrm{~Hz}), 3.8(6 \mathrm{H}, \mathrm{s}$, $\left.-\mathrm{OCH}_{3}\right), 11.7$ (br.s -COOH). m/z (70 ev) (\%) = $208\left(100, \mathrm{M}^{+}\right), 191$ (8.99), 177 (9.87), 163 (15.17), 137 (6.98), 132 (5.21), 107 (3.78), 106 (2.07), 77 (24.43), 75 (3.82), 51 (23.82). Anal. Cald for $\mathrm{C}_{15} \mathrm{H}_{10} \mathrm{O}_{6}$ : C, 63.45; H, 5.81. Found: C, 63.41; H, 5.84\%.

3-(3',5'Dimethoxyphenyl)propionic acid (3). 3,5-Dimethoxycinnamic acid (2) (25g, 0.1mole) was dissolved in IM solution of sodium hydroxide $(93 \mathrm{ml})$ contained in a 500 $\mathrm{ml}$ two-necked flask equipped with a mechanical stirrer and added sodium amalgam (286g) gradually during 1 hour while the mixture is well stirred. When hydrogen was not evolved, the mercury was separated and washed with water. Washing was added to the solution and acidified the whole mixture with dilute hydrochloric acid (1:1). 3-(3',5'-dimethoxyphenyl)propionic acid (3) was precipitated at 
first in the form of oil, which solidified on cooling and rubbing with a glass rod. Filtered at the pump and recrystalized with pet. ether gave 3-(3',5'-dimethoxyphenyl)propionic acid (3) white solid (23g, 0.110mole, 92\%) m.p 58 ${ }^{\circ}$. IR $\left(v_{\max }, \mathrm{KBr}, \mathrm{cm}^{-1}\right)=3400-3190,2950,2835,1722$, 1470, 1364, 1207. ${ }^{1} \mathrm{H}-\mathrm{NMR}\left(\mathrm{CDCl}_{3}, 300 \mathrm{MHz}\right): \delta(\mathrm{ppm})=6.36(2 \mathrm{H}, \mathrm{d}, J=2.3 \mathrm{~Hz}), 6.32(1 \mathrm{H}, \mathrm{t}, J$ $=2.1 \mathrm{~Hz}), 2.89(2 \mathrm{H}, \mathrm{t}, J=8.0 \mathrm{~Hz}), 2.66(2 \mathrm{H}, \mathrm{t}, J=7.5 \mathrm{~Hz}), 3.76\left(6 \mathrm{H}, \mathrm{s},-\mathrm{OCH}_{3}\right), 11.66(\mathrm{br} . \mathrm{s},-$ $\mathrm{COOH}) . \mathrm{m} / \mathrm{z}(70 \mathrm{ev})(\%)=210\left(71, \mathrm{M}^{+}\right), 180$ (1.52), 165 (100), 150 (11.09), 139 (3.27), 105 (30.49), 91 (39.59), 65 (28.80). Anal. Cald for $\mathrm{C}_{11} \mathrm{H}_{14} \mathrm{O}_{4}$ : C, 62.85; H 6.71. Found: C, 62.81; $\mathrm{H}$ 6.73 .

5,7-Dimethoxy-1-indanone (4). 3-(3',5'-Dimethoxyphenyl)propionic acid (3) (23g, 0.1 moles) was dissolved in $250 \mathrm{~g}$ of hot poly phosphoric acid at $80^{\circ} \mathrm{C}$. The resulting yellow solution was heated on oil bath with stirring for 2 hours. The cooled solution was added to ice water $(500 \mathrm{ml})$. The mixture was extracted with ethyl acetate $(3 \times 150)$ and washed the combined extracts with $5 \%$ sodium hydroxide solution and then with water until the washings were neutral. Dried the ethyl acetate solution over magnesium sulphate. The organic layer was concentrated and purified on silica gel using pet. ether $\left(40-80^{\circ} \mathrm{C}\right)$ as eluent to afford 5,7-dimethoxy-1-indanone (3) light yellow crystals $\left(20 \mathrm{~g}, 0.1\right.$ mole, 87\%) m.p $97{ }^{\circ} \mathrm{C}$. IR $\left(v_{\max }, \mathrm{KBr}, \mathrm{cm}^{-1}\right)=2947,1696,1645,1605$, 1215, 1048. ${ }^{1} \mathrm{H}-\mathrm{NMR}\left(\mathrm{CDCl}_{3}, 300 \mathrm{MHz}\right): \delta(\mathrm{ppm})=2.9(2 \mathrm{H}, \mathrm{t}, J=6.2 \mathrm{~Hz}), 2.53(2 \mathrm{H}, \mathrm{t}, J=6.1$ $\mathrm{Hz}), 6.37(1 \mathrm{H}, \mathrm{d}, J=1.4 \mathrm{~Hz}), 6.18(1 \mathrm{H}, \mathrm{d}, J=1.6 \mathrm{~Hz}), 3.77\left(3 \mathrm{H}, \mathrm{s},-\mathrm{OCH}_{3}\right), 3.80\left(3 \mathrm{H}, \mathrm{s}-\mathrm{OCH}_{3}\right)$. $\mathrm{m} / \mathrm{z}(70 \mathrm{ev})(\%)=192\left(96.62, \mathrm{M}^{+}\right), 163(100), 162$ (36.01), 161 (90.11), 150 (3.32) 134 (38.19), 133 (28.90), 131(31.04), 119 (25.62), 105 (33.86), 106 (41.71), 103 (29.96), 75 (16.68). Anal. Cald for $\mathrm{C}_{11} \mathrm{H}_{12} \mathrm{O}_{3}$ : C, 68.74; H, 6.29. Found: C, 68.71; H, 6.30.

Methyl 2-hydroxy-2-(5,7-dimethyoxy-1-oxo-1H-inden-2(3H)-ylidene)acetate (5). Sodium $(15.2 \mathrm{~g})$ was dissolved in methanol $(27.5 \mathrm{~g})$ and benzene $(440 \mathrm{ml})$ was added to this solution. Indanone (4) (20g, 0.1 mole) was added in portions with good stirring, then diethyl oxalate $(38.5 \mathrm{ml})$ was added as single aliquot and the mixture was stirred for over night at room temperature. Hydrochloric acid (5\%) was added under ice cooling to the residue obtained after removal of the solvent, to give a final $\mathrm{pH}$ of 1.0. The precipitate that deposited was collected on a filter, washed with hydrochloric acid (5\%), then with water, and dried. The solid thus obtained was crystallized from methanol to give methyl 2-hydroxy-2-(5,7-dimethyoxy-1-oxo-1H-inden2(3H)-ylidene)acetate (5) as yellow needles (26.5g, 0.09 mole, 91.5\%) ,m.p 234-237 ${ }^{\circ} \mathrm{C}$. IR $\left(v_{\max }, \mathrm{KBr}, \mathrm{cm}^{-1}\right)=3400-2400,1744,1692,1633,1605,1365 .{ }^{1} \mathrm{H}-\mathrm{NMR}\left(\mathrm{CDCl}_{3}, 300 \mathrm{MHz}\right): \delta$ $(\mathrm{ppm})=3.93\left(2 \mathrm{H}, \mathrm{s},-\mathrm{CH}_{2}-\right), 4.01(3 \mathrm{H}, \mathrm{s}, \mathrm{COOMe}), 4.05(6 \mathrm{H}, \mathrm{s}, 2 \times \mathrm{OMe}), 6.38(1 \mathrm{H}, \mathrm{d}, J=3.05$ $\mathrm{Hz}$, arom H), $6.80(1 \mathrm{H}, \mathrm{d}, J=3.0 \mathrm{~Hz}$, arom H), $13.57(\mathrm{~s},-\mathrm{OH}) . \quad) . \mathrm{m} / \mathrm{z}(70 \mathrm{ev})(\%)=278.2$ (19.3, $\mathrm{M}^{+}$), 219.1 (95.11), 191.1 (08.44), 175 (06.33), 85.0 (64.99), 83.0 (100), 42.0 (24.75). Anal. Cald for $\mathrm{C}_{14} \mathrm{H}_{14} \mathrm{O}_{6}$ : C, 60.43; H, 5.07. Found: C, 60.48; H, 5.10.

3,5-Dimethoxyhomophthalic acid (6). A solution of methyl 2-hydroxy-2-(5,7-dimethyoxy-1oxo-1H-inden-2 (3H)-ylidene) acetate (5) (26.5g, $0.1 \mathrm{~mole})$ and potassium hydroxide $(20.8 \mathrm{~g})$ in 
water $(442 \mathrm{ml})$ was cooled to $5-10{ }^{\circ} \mathrm{C}$, and hydrogen peroxide $(90.0 \mathrm{~g}, 35 \%)$ was added. The reaction mixture was stirred at this temperature for 6 hours. When the temperature was gradually raised to $50{ }^{\circ} \mathrm{C}$, vigorous frothing occurred. After stirring for a further $2 \mathrm{~h}$ at this temperature, the reaction mixture was filtered and the filtrate was acidified with concentrated hydrochloric acid. The crystals that precipitated were collected by filtration and the filtrate was extracted with ethyl acetate $(4 \times 30 \mathrm{ml})$. The solvent was evaporated to give solid residue, which was purified by recrystalization from dichloromethane to give 3,5-dimethoxyhomophthalic acid (6) (19g, 0.072 mole, $72 \%)$ as light yellow color micro crystals. m.p $170^{\circ} \mathrm{C}$. IR $\left(v_{\max }, \mathrm{KBr}, \mathrm{cm}^{-1}\right)=2580$ 3300, 1714, 1659,1226. ${ }^{1} \mathrm{H}-\mathrm{NMR}\left(\mathrm{CDCl}_{3}, 300 \mathrm{MHz}\right): \delta(\mathrm{ppm})=6.55(1 \mathrm{H}, \mathrm{d}, J=1.2 \mathrm{~Hz}), 6.84$ $(1 \mathrm{H}, \mathrm{d}, J=1.4 \mathrm{~Hz}), 4.2(2 \mathrm{H}, \mathrm{s}), 3.65\left(3 \mathrm{H}, \mathrm{s},-\mathrm{OCH}_{3}\right), 3.67\left(3 \mathrm{H}, \mathrm{s},-\mathrm{OCH}_{3}\right), 11.7$ (br.s $\left.-\mathrm{COOH}\right)$. ). $\mathrm{m} / \mathrm{z}(70 \mathrm{ev})(\%)=240\left(38.12, \mathrm{M}^{+}\right), 222$ (17.17), 196 (66.44), 195 (40.33), 194 (84.99), 178 (100), 151 (28.75), 150 (15.10), 120 (41.52), 119(12.24). Anal. Cald for $\mathrm{C}_{11} \mathrm{H}_{12} \mathrm{O}_{6}$ : C, 55.00; H, 5.04. Found: C, 54.92; H, 5.11.

3-(3',4'-Dimethoxyphenyl)-6,8-dimethoxyisocoumarin ${ }^{25}$ (9). Mixture of 3,4-dimethoxybenzoic acid (7) $(2 \mathrm{~g}, 10.9 \mathrm{mmol})$ and thionyl chloride $(1.57 \mathrm{~g}, 1 \mathrm{ml}, 13.2 \mathrm{mmol})$ was heated for $30 \mathrm{~min}$ in the presence of a drop of dimethylformamide at $90^{\circ} \mathrm{C}$. Completion of the reaction was indicated by the stoppage of gas evolution. Removal of excess of thionyl chloride was carried out under reduced pressure to afford 3,4-dimethoxybenzoyl chloride (8) (1.8g, 9mmol). 3,5Dimethoxyhomophthalic acid (6) (0.63g, $2.6 \mathrm{mmol})$ and 3,4-dimethoxybenzoyl chloride (8) $(1.8 \mathrm{~g}, 9 \mathrm{mmol})$ was refluxed at $200^{\circ} \mathrm{C}$ for six hours. Residue, after concentration, was purified by HPLC to give the 3-(3',4'-dimethoxyphenyl)-6,8-dimethoxyisocoumarin (9) as yellow solid $(60 \%)$. m.p. $175-177^{\circ} \mathrm{C}$; IR: $\left(v_{\max }, \mathrm{KBr}, \mathrm{cm}^{-1}\right)=1690,1626,1599,1365,1322,1243,1192$, 1163. ${ }^{1} \mathrm{H}-\mathrm{NMR}\left(\mathrm{CDCl}_{3}, 300 \mathrm{MHz}\right): \delta(\mathrm{ppm})=3.79-3.88(12 \mathrm{H}, 4 \times \mathrm{s}), 6.46(1 \mathrm{H}, \mathrm{d}, J=8.7 \mathrm{~Hz}, \mathrm{H}-$ 7), 6.54 (1H, d, $J=1.8 \mathrm{~Hz}, \mathrm{H}-5), 6.99$ (1H, s, H-4), 7.15 (1H, d, J=7.8 Hz, H-5'), 7.27(1H, d, $J$ $\left.=1.8 \mathrm{~Hz}, \mathrm{H}-2^{\prime}\right), 7.32\left(1 \mathrm{H}, \mathrm{dd}, J=1.2,8.4 \mathrm{~Hz}, \mathrm{H}-6{ }^{\prime}\right) ;{ }^{13} \mathrm{C}-\mathrm{NMR}\left(\mathrm{CDCl}_{3}, 300 \mathrm{MHz}\right): \delta(\mathrm{ppm})=$ 55.8-56.3 (4× $\left.\mathrm{OCH}_{3}\right), 111.8(\mathrm{C}-7), 112.2$ (C-5), $112.6(\mathrm{C}-8 \mathrm{a}), 120.5\left(\mathrm{C}-2^{\prime}\right), 123.4\left(\mathrm{C}-5^{\prime}\right), 123.6$ (C-6'), 125.4 (C-1'), 148.7 (C-4a), 149.2 (C-3), 154.9 (C-1, C-3', C-4'), 162.8 (C-8), 167.6 (C-6). $\mathrm{m} / \mathrm{z}(70 \mathrm{eV})(\%)=342(6), 314(30), 298$ (2), 284 (30), 254 (54), 206 (32), 166(7), 164 (100), 138 (21),118 (1), 109 (13). Anal. Cald for $\mathrm{C}_{19} \mathrm{H}_{18} \mathrm{O}_{6}$ : C, 66.66; H, 5.30. Found: C, 66.59; H, 5.33\%.

3-(3',4'-Dihydroxyphenyl)-6,8-dihydroxyisocoumarin (10). Freshly distilled hydro- bromic acid $(55 \%, 16 \mathrm{ml})$ was added to stirred solution of isocoumarin $(9)(0.38 \mathrm{~g}, 1.1 \mathrm{mmol})$ in glacial acetic acid $(16 \mathrm{ml})$. Reaction mixture was then refluxed for four hours, cooled and poured on crushed ice and then treated with solid sodium carbonate till $\mathrm{pH}-7$ and extracted with diethyl ether $(2 \times 30 \mathrm{ml})$, dried $\left(\mathrm{Na}_{2} \mathrm{SO}_{4}\right)$ and concentrated to give crude solid which was purified by HPLC to get 3-(3,4-dihydroxyphenyl)-6,8-dihydroxyisocoumarin (10) as pale yellow solid (53\%). m.p. $247-249^{\circ} \mathrm{C}\left(\right.$ lit. mp $\left.244^{\circ} \mathrm{C}\right)$; IR: $\left(v_{\max }, \mathrm{KBr}, \mathrm{cm}^{-1}\right)=3200-3500,1692,1629,1599$, 1365, 1325, 1243, 1190, 1165. ${ }^{1} \mathrm{H}-\mathrm{NMR}\left(\mathrm{CDCl}_{3}, 300 \mathrm{MHz}\right): \delta(\mathrm{ppm})=6.36(1 \mathrm{H}, \mathrm{d}, J=8.7 \mathrm{~Hz}$, H-7), 6.49 (1H, d, $J=1.8 \mathrm{~Hz}, \mathrm{H}-5), 6.89(1 \mathrm{H}, \mathrm{s}, \mathrm{H}-4), 6.05$ (1H, d, $\left.J=1.8 \mathrm{~Hz}, \mathrm{H}-5^{\prime}\right), 6.19(1 \mathrm{H}$, $\left.\mathrm{d}, J=7.9 \mathrm{~Hz}, \mathrm{H}-2^{\prime}\right), 7.26\left(1 \mathrm{H}, \mathrm{dd}, J=1.2,8.4 \mathrm{~Hz}, \mathrm{H}-6{ }^{\prime}\right) .{ }^{13} \mathrm{C}-\mathrm{NMR}\left(\mathrm{CDCl}_{3}, 300 \mathrm{MHz}\right): \delta(\mathrm{ppm})$ =113.8 (C-7), 115.2 (C-5), 112.6 (C-8a), 122.5 (C-2'), $125.4\left(\mathrm{C}-5^{\prime}\right), 124.6\left(\mathrm{C}-6^{\prime}\right), 126.4\left(\mathrm{C}-1^{\prime}\right)$, 
148.7 (C-4a), 147.2 (C-3), 151.9 (C-1, C-3', C-4'), 163.8 (C-8), $168.6(\mathrm{C}-6) . \mathrm{m} / \mathrm{z}(70 \mathrm{eV})(\%)=$ 286 (7), 244 (4), 259 (52), 243(27), 227 (66), 178(31), 150(1),136 (100),138 (7), 110(32), 94(13). Anal. Cald for $\mathrm{C}_{15} \mathrm{H}_{10} \mathrm{O}_{6}$ : C, 62.94; H, 3.52. Found: C, 62.99; H, 3.44\%.

\section{References}

1. Matsuda, H.; Shimoda, H.; Yoshikawa, M. Bioorg. Med. Chem. 1999, 7, 1445.

2. Yoshikawa, M.; Harada, E.; Naitoh, Y.; Inoue, K.; Matsuda, H.; Shimoda, H.; Yamahara, J.; Murakami, N. Chem. Pharm. Bull. 1994, 42, 2225.

3. (a) Matsuda, H.; Shimoda, H.; Yamahara, J.; Yoshikawa, M. Bioorg. Med. Chem. Lett. 1998, 8, 215. (b) Shimoda, H.; Matsuda, H.; Yamahara, J.; Yoshikawa, M. Biol. Pharm. Bull. 1998, $21,809$.

4. Whyte, A. C.; Gloer, J. B.; Scott, J. A.; Mallock, D. J. Nat. Prod. 1996, 59, 765.

5. Nozawa, K.; Yamada, M.; Tsuda, Y.; Kawai, K.; Nakajima, S. Chem. Pharm. Bull. 1981, 29, 2689.

6. Furuta, T.; Fukuyama, Y.; Asakawa, Y. Phytochemistry 1986, 25, 517.

7. Lee, J. H.; Park, Y. J.; Kim, H. S.; Hong, Y. S.; Kim, K. W.; Lee, J. J. J. Antibiot. 2001, 54, 463.

8. Hedge, V. R.; Wittreich, H.; Patel, M. G.; Horan, A. C.; Hart, R. F.; Troyanovich, J. J.; Puar, M. S.; Gullo, V. P. J. Ind. Microbiol. 1989, 4, 209.

9. Umehara, K.; Matsumoto, M.; Nakamura, M.; Miyase, T.; Kuronyanagi, M.; Noguchi, H. Chem. Pharm. Bull. 2000, 48, 566.

10. (a) Barry, R. D. Chem. Rev. 1964, 64, 229. (b) Houser, F. M.; Baghdanov, V. M. J. Org. Chem. 1988, 53, 4676. (c) Mali, R. S.; Babu, K. N. J. Org. Chem. 1998, 63, 2488.

11. (a) Jones, J. B.; Pinder, A. R. J. Chem. Soc. 1958, 2612. (b) Tirodkar, R. B.; Usgaonkar, R. N. Indian J. Chem. 1972, 10, 1060.

12. For a review, see: Napolitano, E. Org. Prep. Proced. Int. 1997, 29, 631.

13. (a) Izumi, T.; Morishita, N. J. Heterocycl. Chem. 1994, 31, 145. (b) Ohta, S.; Kamata, Y.; Inagaki, T.; Masuda, Y.; Yamamoto, S.; Yamashita, M.; Kawasaki, I. Chem. Pharm. Bull. 1993, 41, 1188. (c) Yoshikawa, M.; Harada, E.; Yagi, N.; Okuno, Y.; Muraoka, O.; Aoyama, H.; Murakami, N. Chem. Pharm. Bull. 1994, 42, 721. (d) Yoshikawa, M.; Shimada, H.; Yagi, N.; Murakami, N.; Shimoda, H.; Yamahara, J.; Matsuda, H. Chem. Pharm. Bull. 1996, 44, 1890. (f) Rama, N. H.; Iqbal, R.; Zamani, K.; Saeed, A.; Iqbaq1, M. Z.; Choudhary, M. I. Indian J. Chem. Sect. B 1998, 37, 365. (g) Bovicelli, P.; Lupattelli, P.; Crescenzi, B.; Sanetti, A.; Bernini, R. Tetrahedron. 1999, 55, 14719. (h) Mal, D.; Bandyopadhyay, M.; Ghorai, S. K.; Datta, K. Tetrahedron Lett. 2000, 41, 3677. (i) Hussain, M. T.; Rama, N. H.; Malik, A. Indian J. Chem. Sect. B. 2001, 40, 372.

14. (a) Minami, T.; Nishimoto, A.; Nakamura, M. Chem. Pharm. Bull. 1994, 42, 1700. (b) Sashida, H.; Kawamukai, A. Synthesis. 1999, 1145. (c) Wang, L.; Shen, W. Tetrahedron 
Lett. 1998, 39, 7625. (d) Liao, H. Y.; Cheng, C. H. J. Org. Chem. 1995, 60, 3711. (e) Bellina, F.; Ciucci, D.; Vergamini, P. G.; Rossi, R. Tetrahedron. 2000, 56, 2533.

15. (a) Chatterjea, J. N.; Mukherjee, S. K.; Bhakta, C.; Jha, H. C.; Zilliken, F. Chem. Ber. 1980, 113, 3927. (b) Nagarajan, A.; Balasubramanian, T. R. Indian J. Chem. Sect. B 1988, 27, 380. (c) Natsugari, H.; Ikeura, Y.; Kiyota, Y.; Ishichi, Y.; Ishimaru, T.; Saga, O.; Shirafuji, H.; Tanaka, T.; Kamo, T.; Doi, T.; Otsuka, M. J. Med. Chem. 1995, 38, 3106. (d) Larock, R. C.; Doty, M. J.; Han, X. J. Org. Chem. 1999, 64, 8770.

16. Yoshikawa, M.; Uchida, E.; Chatani, N.; Murakami, N.; Yamahara, J. Chem. Pharm. Bull. 1992, 40, 3121.

17. Yoshikawa, M.; Uchida, E.; Chatani, N.; Kobayashi, H.; Naitoh, Y.; Okuno Y.; Matsuda, H.; Yamahara, J.; Murakami, N. Chem. Pharm.Bull. 1992, 40, 3352.

18. Yoshikawa, M.; Harada, E.; Naitoh, Y.; Inoue, K.; Matsuda, H.; Shimoda, H.; Yamahara, J.; Murakami, N. Chem. Pharm. Bull. 1994, 42, 2225.

19. Yoshikawa, M.; Matsuda, H.; Shimoda, H.; Shimada, H.; Harada, E.; Naitoh, Y.; Miki, A.; Yamahara, J.; Murakami, N. Chem. Pharm. Bull. 1996, 44, 1440.

20. Matsuda, H.; Shimoda, H.; Yoshikawa, M. Bioorg. Med. Chem. 1999, 7, 1445.

21. Rossi, R.; Carpita, A.; Bellina, F.; Stabile, P.; Mannina, L. Tetrahedron 2003, 592067.

22. Takeuchi, N.; Murase, M.; Ochi, K.; Tobinaga, S. J. Chem. Soc., Chem. Comm. 1980, 13, 593.

23. Qadeer. G.; Rama, N. H.; Zhijin, F. Chem. J. Chinese Uni. 2007(In press).

24. Bauta, W. E.; Lovett, D. P.; Cantrell, W. R.; Bruke, B. D. J. Org. Chem. 2003, 68, 5967.

25. Khan, K. M.; Ahmed, S.; Khan, Z. A.; Rani, M.; Perveen, S.; Choudhary, M. I.; Rahman, A. Lett. Org. Chem. 2005, 2, 532. 